\title{
Non-invasive measurements of atherosclerosis in adult cystinosis patients
}

\author{
Martine T. P. Besouw • Suzanne Holewijn • \\ Elena N. Levtchenko • Mirian C. H. Janssen
}

Received: 3 November 2010 /Revised: 28 December 2010 / Accepted: 13 January 2011 /Published online: 9 February 2011

(C) The Author(s) 2011. This article is published with open access at Springerlink.com

\begin{abstract}
Background Cystinosis is characterized by intralysosomal cystine accumulation, causing end stage renal disease around 10 years of age if not treated with cysteamine. Cystine accumulation in blood vessels might increase atheroma formation or arterial stiffness and therefore increase the risk for cardiovascular disease (CVD). This study aimed to investigate the risk for CVD by noninvasive measures of atherosclerosis (NIMA) and to evaluate the effect of cysteamine treatment.

Patients and methods Thirteen Dutch adult cystinosis patients were included. White blood cell (WBC) cystine levels, glomerular filtration rate (GFR) and concommitant medications were obtained from medical records. NIMA included carotid intima-media thickness (cIMT, $\mathrm{n}=13$ ), pulse wave velocity (PWV, $\mathrm{n}=8$ ) and pulse wave analysis (PWA, $\mathrm{n}=6$ ).

Results GFR ranged between $4-95 \mathrm{~mL} / \mathrm{min} / 1.73 \mathrm{~m}^{2}$. All but one patient were treated with cysteamine, mean WBC cystine values ranged between $0.34-1.64 \mathrm{nmol}$ cystine/mg protein, 8 patients had mean WBC cystine levels $<1 \mathrm{nmol}$ cystine/mg protein. When compared to healthy subjects, cIMT and PWV levels were above normal values in 1
\end{abstract}

Communicated by: K. Michael Gibson

Competing interest: None declared

M. T. P. Besouw $\cdot$ E. N. Levtchenko

Department of Pediatric Nephrology,

University Hospitals Leuven,

Leuven, Belgium

S. Holewijn • M. C. H. Janssen $(\bowtie)$

Department of Internal Medicine,

Radboud University Medical Centre,

463 PO box 9101, $6500 \mathrm{HB}$, Nijmegen, The Netherlands

e-mail: M.Janssen@aig.umcn.nl patient for each measure. PWA measurements showed high augmentation index in three patients who did not receive lipid-lowering medication. When corrected for renal function, cIMT and PWV levels were within the normal range. Conclusion Young adult cystinosis patients treated with cysteamine have no additional risk for CVD when compared to patients with chronic kidney disease of other causes.

\section{Abbreviations \\ CVD Cardiovascular disease \\ CKD Chronic kidney disease \\ NIMA Non-invasive measures of atherosclerosis \\ cIMT Carotid intima-media thickness \\ PWV Pulse wave velocity \\ PWA Pulse wave analysis \\ AI Augmentation index \\ SEVR Sub-endocardial viability ratio}

\section{Introduction}

Cystinosis is a metabolic disorder caused by mutations in the CTNS gene, encoding the lysosomal cystine carrier cystinosin. This results in intralysosomal accumulation of the amino acid cystine (Town et al. 1998). The disease is inherited in an autosomal recessive trait and has an incidence of one case in every 100,000 to 200,000 live births. Most children present during the first year of life with generalized proximal tubular dysfunction called renal Fanconi syndrome. If left untreated, the disease progresses towards end-stage renal disease around the age of 10 years. Besides renal involvement, the eyes, endocrine organs, muscles and central nervous system are also affected. Cystinosis is treated by administration of the cystine- 
depleting agent cysteamine, which slows down the progression of renal failure and protects extra-renal organs (Gahl et al. 2002).

Prior to renal transplantation and cysteamine treatment, the lifespan for children with cystinosis was approximately 10 years. Now cystinosis patients have survived through their fifth decade, but the unremitting accumulation of cystine has created significant extra-renal morbidity and mortality. Ueda et al. found increased coronary and vascular calcifications in cystinosis patients and hypothesized that the accumulation of intracellular cystine itself may be a risk factor for vascular calcifications. They advised to screen older patients with cystinosis for this complication (Ueda et al. 2006). We hypothesize that cystine accumulation in blood vessels might increase atheroma formation or arterial stiffness and therefore increase the risk for cardiovascular disease CVD. Furthermore, it has been reported that patients with cystinosis might be prone to hypercholesterolemia (Nesterova and Gahl 2008, Murphy and Papathakis 1993). High cholesterol levels might further add to the risk for cardiovascular disease (CVD) in cystinosis patients.

Next to the possible deteriorative effect of cystine accumulation in blood vessels and hypercholesterolemia, patients with cystinosis can be prone to CVD due to chronic kidney disease (CKD) (Go et al. 2004). Whether cysteamine treatment offers a protection against cardiovascular damage remains unclear.

Non-invasive measurements of atherosclerosis (NIMA) have been well established as an indicator for atherosclerosis and may be used as a predictor for the development of CVD. In this study we focussed on two aspects of atherosclerosis measured by three NIMA in adult cystinosis patients, in order to investigate the risk for CVD in this population. These three NIMA include carotid intima-media thickness (cIMT), which reflects the atheroma part of the atherosclerotic process, pulse wave velocity (PWV) and pulse wave analysis (PWA), which both reflect arterial stiffness.

\section{Patients and methods}

All patients $\geq 21$ years old who were treated for cystinosis in the Netherlands were included in this study. A total of 13 patients met these criteria, seven of whom were male. Mutation analysis of the CTNS gene was performed in 12 patients and confirmed the diagnosis of cystinosis in all of them, genetic analysis was not performed in one patient. White blood cell (WBC) cystine levels, measured at least annually during the last 5 years (range 5-18 years), were obtained from the patients' medical records. These values were used to calculate mean WBC cystine levels. Patients were considered adequately treated with cysteamine if their mean WBC cystine level was below 1 nmol cystine/mg protein. All patients had taken cysteamine in the past, at the moment of this study it was still taken by $12 / 13$ patients. Glomerular filtration rate (GFR) was calculated using the modification of diet in renal disease study equation (MDRD) (Levey et al. 1999). Hypertension was defined as a $\mathrm{BP}$ of $>140 / 90 \mathrm{mmHg}$ or taking medication for high BP (K/DOQI 2004).

Measurements of cIMT, PWV and PWA were performed according to a highly standardized protocol and performed by the same experienced technician in all patients (Holewijn et al. 2009). Mean cIMT was calculated from the mean of the following four measured segments: far wall left, far wall right, near wall left and near wall right. Subsequently the presence of plaque and its thickness was measured. Plaque was defined as focal thickening of the wall of at least $1.5 \mathrm{x}$ mean cIMT.

PWV and PWA were measured with applanation tonometry, using the commercially available SphygmoCor system version 7.1 (Atcor Medical, Sydney. Australia). PWV was acquired in 8/13 subjects. Due to logistic reasons, measurements of arterial stiffness could not be performed in all patients. In two male patients PWA could not be recorded, since both had a history of multiple shunt surgeries in both arms. Therefore, PWA measurements were acquired in 6/13 patients. Three parameters of PWA were analyzed; the augmentation index (AI), the ejection duration and the Buckberg sub-endocardial viability ratio index (Buckberg SEVR index). Since the AI strongly depends on heart rate (Wilkinson et al. 2000), it was corrected for a heart rate of 75 beats per minute.

\section{Results}

Clinical and biochemical characteristics of the included patients are depicted in Table 1. Median age was 28 (range 21-44) years, the female patients were generally older compared to the male patients. GFR ranged between 4$95 \mathrm{~mL} / \mathrm{min} / 1.73 \mathrm{~m}^{2}$. All patients underwent a renal transplantation in the past, 12 of them still had functioning renal graft at the time of this study, one male patient was treated with hemodialysis after graft failure. Median time after renal transplantation was 14 (range 1-29) years. Twelve patients were receiving anti-hypertensive drugs. Eight patients were adequately treated with cysteamine, proven by mean WBC cystine levels $<1 \mathrm{nmol}$ cystine/mg protein. Five patients ( 3 male, 2 female) were taking lipidlowering medication. Only one female patient, not taking lipid-lowering medication, had a serum low density lipoprotein (LDL) cholesterol higher than $3.5 \mathrm{mmol} / \mathrm{L}$. All patients had a LDL/HDL ratio below five. 


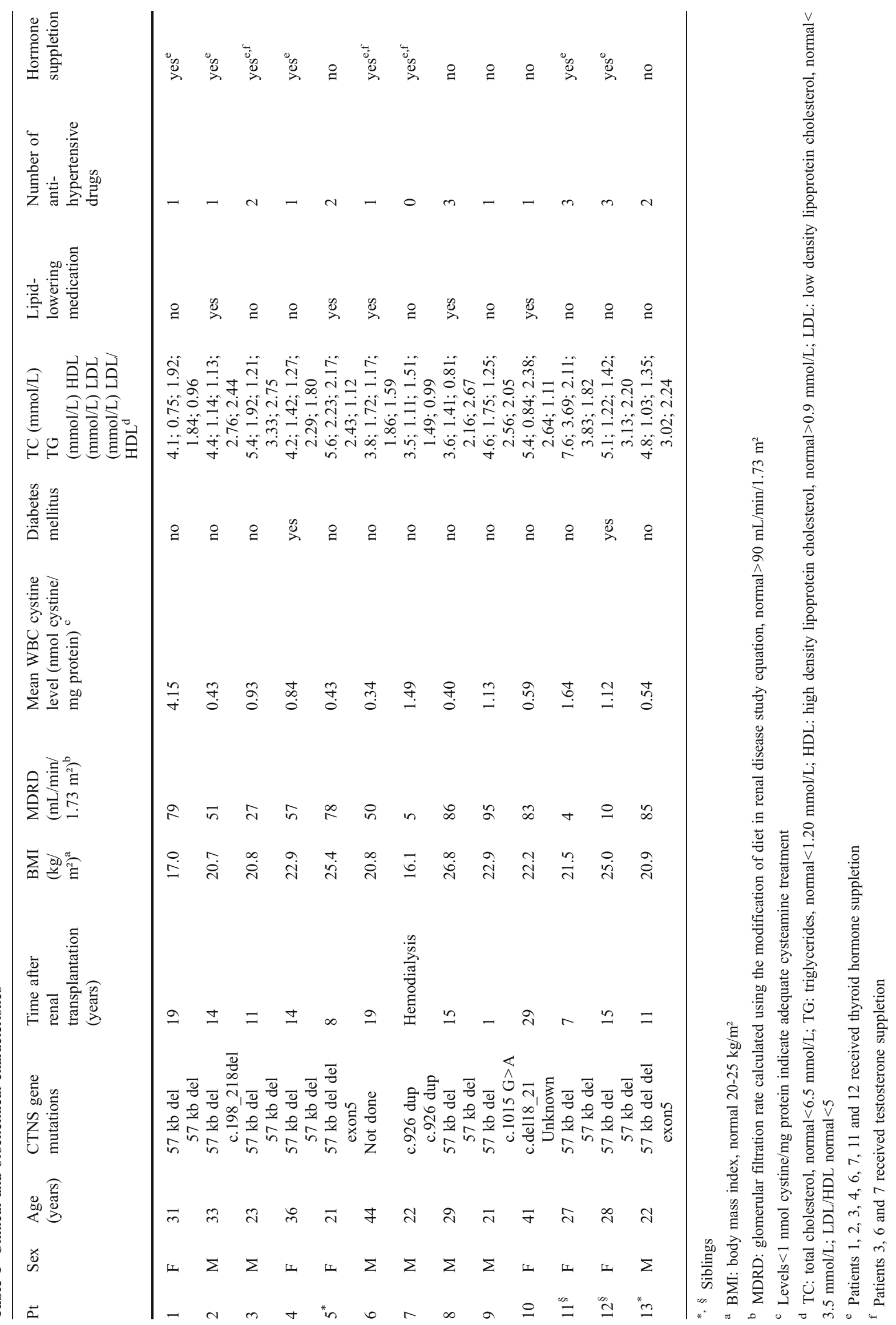


Non-invasive measurements of atherosclerosis

The results of cIMT, PWV, and PWA measurements are depicted in Table 2 and will be subsequently described in the sections below.

\section{cIMT measurements}

The cIMT measurements were performed in all 13 patients. Median cIMT was 0.64 (range 0.54-0.82) $\mathrm{mm}$. In none of the patients plaques were detected at the carotid arteries. When compared to a healthy population of the same age (normal range $\pm 2 \mathrm{SD}$ : men $0.39-0.79 \mathrm{~mm}$, women 0.41-0.73 mm, $n=2265$ ) (Juonala et al. 2008), mean cIMT values were more than $2 \mathrm{SD}$ above the expected mean in only 1 female with CKD stage 3 (patient 3 ), who had a functional renal graft since 14 years and was adequately treated with cysteamine. However, when corrected for CKD stage (Hogg et al. 2003), cIMT levels were within or even below the normal range in all patients (Fig. 1a) (Yilmaz et al. 2010).

\section{PWV measurements}

PWV measurements were performed in eight patients, five of whom were male. Men had higher PWV levels compared to women, with a median value of 7.2 (range 5.6-9.4) $\mathrm{m} / \mathrm{s}$ and 6.1 (range 4.8-7.5) $\mathrm{m} / \mathrm{s}$, respectively. When compared to healthy subjects of the same age, one male patient (patient 8) had a PWV value of more than 2 SD above the expected mean (normal range $\pm 2 \mathrm{SD}: 20-29$ years $4.7-7.6 \mathrm{~m} / \mathrm{s}, 30-39$ years $3.8-9.2 \mathrm{~m} / \mathrm{s}, 40-49$ years $4.6-9.8 \mathrm{~m} / \mathrm{s}, n=1455$ ) (Mattace-Raso et al. 2010). The patient with a high PWV value had CKD stage 2 and had a functional renal graft since 15 years, he was adequately treated with cysteamine. When corrected for CKD stage (Hogg et al. 2003), all PWV levels were within a range of $2 \mathrm{SD}$ of the expected mean (Fig. 1b) (Wang et al. 2005).

\section{PWA measurements}

PWA measurements were performed in six patients (three of whom were male). Since there are no reference values available from literature for CKD patients with an age comparable to our population, the measured values were compared to the normal ranges provided by the manufacturer of the program. The median AI was $25 \%$ (range $2 \%-41 \%$ ). Normal values depend on age, they should be below $15 \%$ at 30 years and below $22 \%$ at 40 years. Three patients had values above the normal range ( 2 female, 1 male). The ejection duration remained within the normal range of $250-350 \mathrm{~ms}$ in all patients. The median Buckberg SEVR index was 176\% (range 164\%-204\%). All patients had a Buckberg SEVR index above $150 \%$, indicating that myocardial perfusion was not reduced.

Table 2 Results of non-invasive measurements of atherosclerosis

\begin{tabular}{|c|c|c|c|c|c|c|c|c|}
\hline $\mathrm{Pt}$ & Sex & $\begin{array}{l}\text { Age } \\
\text { (years) }\end{array}$ & $\begin{array}{l}\text { Blood pressure } \\
(\mathrm{mmHg})\end{array}$ & $\begin{array}{l}\text { Mean cIMT } \\
(\mathrm{mm})^{\mathrm{a}}\end{array}$ & $\begin{array}{l}\mathrm{PWV} \\
(\mathrm{m} / \mathrm{s})^{\mathrm{b}}\end{array}$ & $\begin{array}{l}\mathrm{AI} \\
(\%)^{\mathrm{c}}\end{array}$ & $\begin{array}{l}\mathrm{ED} \\
(\mathrm{ms})^{\mathrm{d}}\end{array}$ & $\begin{array}{l}\text { Buckberg SEVR index } \\
(\%)^{\mathrm{e}}\end{array}$ \\
\hline 1 & $\mathrm{~F}$ & 31 & $132 / 82$ & 0.65 & 7.5 & 41 & 277 & 173 \\
\hline 2 & M & 33 & $116 / 70$ & 0.64 & 5.6 & 14 & 349 & 164 \\
\hline 3 & M & 23 & $102 / 68$ & 0.66 & 6.0 & 30 & 295 & 188 \\
\hline 4 & $\mathrm{~F}$ & 36 & $120 / 74$ & 0.81 & 6.1 & 40 & 343 & 171 \\
\hline $5^{*}$ & $\mathrm{~F}$ & 21 & $119 / 82$ & 0.62 & 4.8 & 2 & 313 & 179 \\
\hline 6 & M & 44 & $116 / 84$ & 0.63 & 9.4 & 20 & 337 & 204 \\
\hline 7 & M & 22 & $112 / 72$ & 0.71 & 7.2 & & & \\
\hline 8 & M & 29 & $128 / 84$ & 0.60 & 8.4 & & & \\
\hline 9 & M & 21 & $120 / 75$ & 0.60 & & & & \\
\hline 10 & $\mathrm{~F}$ & 41 & $135 / 90$ & 0.68 & & & & \\
\hline $11^{\S}$ & $\mathrm{F}$ & 27 & $148 / 100$ & 0.60 & & & & \\
\hline $12^{\S}$ & $\mathrm{F}$ & 28 & $152 / 92$ & 0.64 & & & & \\
\hline $13^{*}$ & M & 22 & $140 / 68$ & 0.54 & & & & \\
\hline
\end{tabular}

\footnotetext{
*, § Siblings

a cIMT: carotid intima-media thickness, normal values depend on age and renal function (see text)

b PWV: pulse wave velocity, normal values depend on age and renal function (see text)

c AI: augmentation index after correction for a heart rate of 75 beats/minute, normal values $<15 \%$ at 30 years, $<22 \%$ at 40 years

${ }^{d}$ ED: Ejection duration, normal 250-350 ms

e SEVR: sub-endocardial viability ratio, normal $>150 \%$
} 


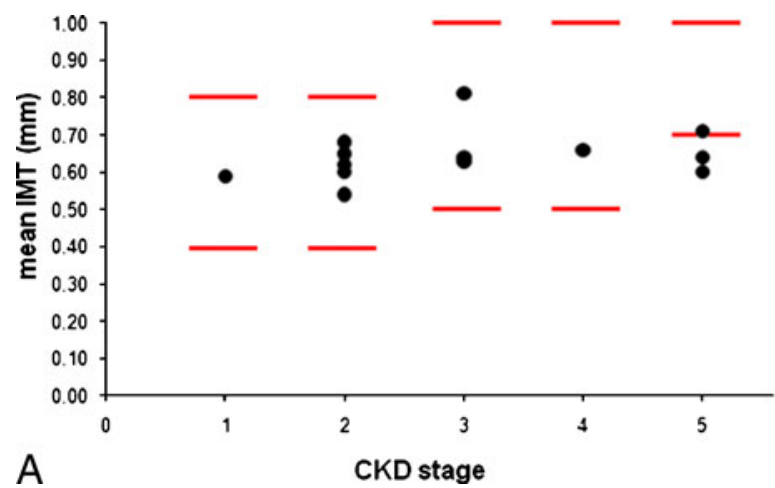

Fig. 1 cIMT and PWV values classified by CKD stage. a: Mean total cIMT values classified by CKD stage. b: PWV classified by CKD stage. The bars indicate the normal range for that particular CKD

\section{Influence of therapy on NIMA}

To evaluate a possible effect of both adequate treatment of cystinosis (defined as mean WBC cystine levels $<1$ nmol cystine/mg protein) and the administration of lipid-lowering medication on NIMA, we displayed our results in function of the patients' treatments. Figure 2a-c show NIMA values in patients with mean WBC cystine levels either below or above $1 \mathrm{nmol}$ cystine/mg protein, Fig. 2e-f shows NIMA values in patients either on or off lipid-lowering medication. In our study population neither mean WBC cystine levels, nor lipid-lowering medication had an effect on cIMT levels (Fig. 2a and d) or PWV values (Fig. 2b and e). PWA

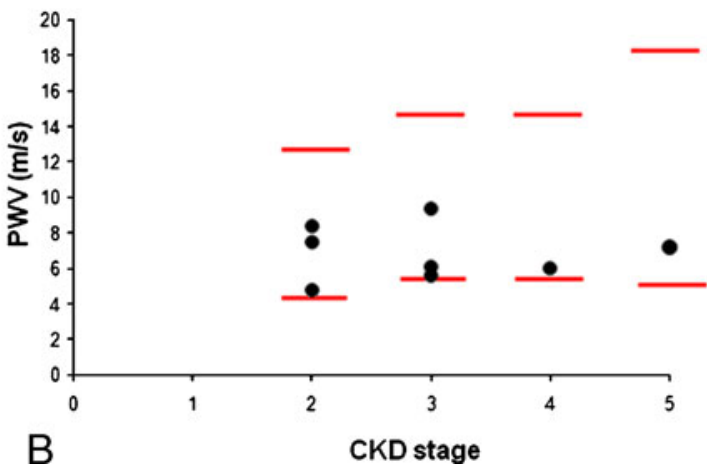

stage. Both cIMT and PWV values are within or below the normal range when corrected for CKD stage

measurements were conducted in only 1 patient with high WBC cystine levels (patient 1), she showed a clearly increased AI (Fig. 2c). None of the three patients with an increased AI was taking lipid-lowering medication (Fig. 2f).

\section{Discussion}

Since increasing numbers of individuals with cystinosis are receiving renal transplants and live into adulthood, more long-term complications of the disease are being recognized. Most likely, the full spectrum of extra-renal
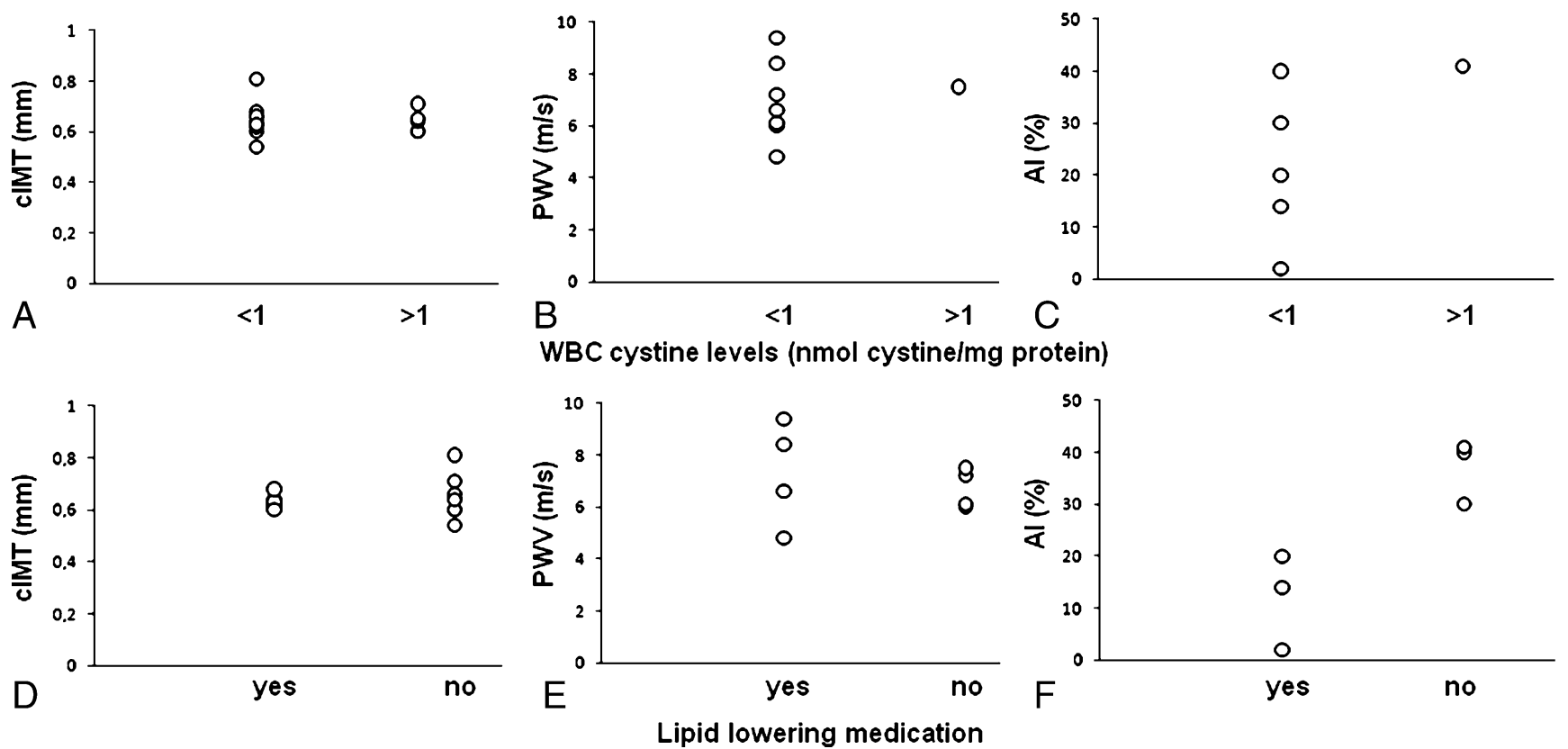

Fig. 2 Effect of cysteamine and lipid-lowering medication on NIMA. Effect of adequate cysteamine treatment, indicated by mean WBC cystine levels $<1$ nmol cystine/mg protein, on cIMT (a), PWV (b) and AI (c). There is no clear effect of WBC cystine levels on cIMT. PWV and PWA measurements were conducted in only one patient with high WBC cystine levels, she showed a clearly increased AI. Effect of lipidlowering medication on cIMT (d), PWV (e) and AI (f). There is no clear difference between cIMT and PWV values of patients who take lipid-lowering medication, compared to those who do not. The three patients with an increased AI did not take any lipid-lowering medication 
involvement in cystinosis has not yet been elucidated, since many of the long-term complications were recognized only after renal transplantation allowing patients to survive past adolescence. Ueda et al. added arteriopathy to the list of long-term complications of cystinosis. Their conclusion was based on the fact that they found increased vascular calcifications on routine chest and head computed tomography (CT) scans (Ueda et al. 2006).

To our knowledge, we for the first time investigated the risk for CVD by three NIMA in cystinosis patients, including cIMT, PWV and PWA, allowing a thorough risk evaluation for CVD in this population. The most important finding of this study is that we found normal NIMA in adult cystinosis patients with extended comorbidity such as diabetes, hypertension and renal failure, when correction for CKD stage was made. The main limitation of this study is the small study population, since cystinosis is a very rare disease. Secondly, we did not have a control group of patients with CKD without cystinosis, but we used the studies of Yilmaz et al. and Wang et al. to compare our results (Yilmaz et al. 2010, Wang et al. 2005).

\section{Non-invasive measures of atherosclerosis}

An increased cIMT may reflect hypertrophy of the intimal and/or medial layer of the carotid arteries, which can be caused by changes in flow, wall tension or lumen diameter (Stein et al. 2008). Since the outcome of cIMT measurements is influenced by renal function, we compared our data to those measured in patients with different stages of CKD (Yilmaz et al. 2010) and found no increase of cIMT values in our study population when compared with patients with the same CKD stage without cystinosis. On the contrary, in cystinosis patients with CKD stage five we found cIMT values that were lower than one would expect according to their renal function (Fig. 1). However, when compared to a healthy population of the same age, one patient had a mean cIMT level more than 2 SD above the expected mean which could not be explained by fluid overload.

PWV reflects the degree of arterial stiffness. Overall, men had higher PWV values than women contrasting other studied demonstrating no differences in PWV related to gender (Smulyan et al. 2001). There was no clear difference between cIMT values, nor was there a difference between blood pressure values between men and women (Table 2). Since our study population was rather small, no conclusion can be drawn from this observation.

In 2005, Wang et al. published PWV values for a large group of CKD patients. Their study population was older than ours, but they used the same equipment to perform their measurements as we did (Wang et al.
2005). Mourad et al. also investigated PWV values in younger CKD patients, however, they used the Complior device to perform their measurements (Mourad et al. 2001). This makes their results more difficult to compare with ours, since both techniques are not interchangeable (Jatoi et al. 2009). Therefore, we compared our values to those published by Wang et al. All PWV values measured in our patients were within $2 \mathrm{SD}$ of the expected mean for the corresponding CKD stage. However, PWV values were more than $2 \mathrm{SD}$ above the expected mean in one patient when compared to healthy subjects of the same age.

The correlation between PWA and cIMT in CKD patients has been well documented (DeLoach et al. 2009). PWA measures can be divided into three subgroups: timing parameters (e.g. heart rate, ejection duration), pressure parameters (e.g. peripheral diastolic and systolic blood pressure, central diastolic and systolic blood pressure) and central aortic indeces (e.g. AI, Buckberg SEVR index). Together they estimate the reflection of the arterial wave in the central circulation. In this study, we foccussed on three measures of PWA, including AI, ejection duration and the Buckberg SEVR index. In our study population we found an increased AI in 3/6 patients, two of which were female. In adults the AI is generally higher in females than in males (Yasmin and Brown 1999). We found no reflection on myocardial perfusion as measured by the Buckberg SEVR index, which was within the normal range in all patients. Overall, there were no clear abnormalities of PWA values in our study population.

The fact that we found low values for cIMT in some patients when corrected for CKD stage, remains interesting. This could be partially explained by the differences in age between our study population and the study population used to generate the reference values. Furthermore, different kinds of medication were used, which could have interfered with the outcomes of the measurements. Most of our patients were treated with antihypertensive drugs and/or lipid-lowering medication starting from an early age, both of which are known to positively influence NIMA (Simon et al. 2002). It should be noted that when compared to healthy subjects of the same age, some patients showed rather high cIMT and PWV levels, reflecting increased stages of the atherosclerotic process, suggesting an increased risk for CVD.

\section{Influence of therapy on NIMA}

It is currently unknown whether cysteamine, which is used to treat cystinosis, may influence NIMA. In this study, we found no differences between cIMT values in patients who were adequately treated with cysteamine 
(WBC cystine levels $<1 \mathrm{nmol} / \mathrm{mg}$ protein) and those who were not (Fig. 2a), however, the group of patients who were less well treated was small $(n=5)$. The only patient who was not treated with cysteamine at the moment of this study, did receive cysteamine treatment in the past. Measurements of PWV and PWA conducted in this patient demonstrated normal PWV values (Fig. 2b), but her AI was increased (Fig. 2c). However, the fact that this observation was made in only one patient makes it impossible to draw conclusions from these results. On the other hand, the fact that most patients had lower NIMA values than one would expect according to CKD stage and the fact that all patients but one were taking cysteamine, might indicate a protective effect of cysteamine. NIMA measurements in a less adequately treated cystinosis population are required to test this hypothesis. It should be stressed out that Ueda et al. found a strong correlation between the number of years without adequate cysteamine therapy and the appearance of vascular calcifications on CT (Ueda et al. 2006). We were not able to determine years on and off cysteamine therapy, since most patients were treated in more than one hospital and several patients had been on and off therapy over the last years. However, all WBC cystine measurements were conducted in our laboratory, independent of the hospital of clinical followup. Therefore, we chose to use mean WBC levels as a measure of cysteamine treatment.

In contrast to previous reports (Nesterova and Gahl 2008, Murphy and Papathakis 1993) we found no large abnormalities in lipid status in our study population (Table 1), which might be due to treatment with lipidlowering medicaton. However, we did not observe a difference in cIMT and PWV values between patients who were taking lipid-lowering medication and those who did not (Fig. 2d and e). The three patients with an increased AI, however, were all not taking lipid-lowering medication (Fig. 2f). This increased AI did not have a reflection on myocardial perfusion, since all patiens had a normal Buckberg SEVR index. These findings are in line with the study of Ueda et al., in which no difference in cholesterol parameters was found between cystinosis patients with and without vascular calcifications on CT (Ueda et al. 2006).

\section{Conclusion}

In conclusion, we demonstrate no increased risk for CVD in young adult cystinosis patients treated with cysteamine. Attention should be paid to long-term follow-up of these patients, including changes of NIMA over time, as it can provide more insight in the possible development of vascular damage in this population.
Open Access This article is distributed under the terms of the Creative Commons Attribution Noncommercial License which permits any noncommercial use, distribution, and reproduction in any medium, provided the original author(s) and source are credited.

\section{Reference}

DeLoach SS, Appel LJ, Chen J et al. (2009) Aortic pulse pressure is associated with carotid IMT in chronic kidney disease: report from Chronic Renal Insufficiency Cohort. Am J Hypertens 22:1235-1241

Gahl WA, Thoene JG, Schneider JA (2002) Cystinosis. N Engl J Med 347:111-121

Go AS, Chertow GM, Fan D, McCulloch CE, Hsu CY (2004) Chronic kidney disease and the risks of death, cardiovascular events, and hospitalization. N Engl J Med 351:1296-1305

Hogg RJ, Furth S, Lemley KV et al. (2003) National Kidney Foundation's Kidney Disease Outcomes Quality Initiative clinical practice guidelines for chronic kidney disease in children and adolescents: evaluation, classification, and stratification. Pediatrics 111:1416-1421

Holewijn S, den Heijer M, Swinkels DW, Stalenhoef AF, de Graaf J (2009) The metabolic syndrome and its traits as risk factors for subclinical atherosclerosis. J Clin Endocrinol Metab 94:28932899

Jatoi NA, Mahmud A, Bennett K, Feely J (2009) Assessment of arterial stiffness in hypertension: comparison of oscillometric (Arteriograph), piezoelectronic (Complior) and tonometric (SphygmoCor) techniques. J Hypertens 27:2186-2191

Juonala M, Kahonen M, Laitinen T et al. (2008) Effect of age and sex on carotid intima-media thickness, elasticity and brachial endothelial function in healthy adults: the cardiovascular risk in Young Finns Study. Eur Heart J 29:1198-1206

Kidney Disease Outcomes Quality Initiative (K/DOQI) (2004) K/ DOQI clinical practice guidelines on hypertension and antihypertensive agents in chronic kidney disease. Am J Kidney Dis 43: S1-290

Levey AS, Bosch JP, Lewis JB, Greene T, Rogers N, Roth D (1999) A more accurate method to estimate glomerular filtration rate from serum creatinine: a new prediction equation. Modification of Diet in Renal Disease Study Group. Ann Intern Med 130:461-470

Mattace-Raso FU, Hofman A, Verwoert GC et al. (2010) Determinants of pulse wave velocity in healthy people and in the presence of cardiovascular risk factors: 'establishing normal and reference values'. Eur Heart J 31:2338-2350

Mourad JJ, Pannier B, Blacher J et al. (2001) Creatinine clearance, pulse wave velocity, carotid compliance and essential hypertension. Kidney Int 59:1834-1841

Murphy JL, Papathakis PC (1993) Hypercholesterolemia in children with cystinosis. Pediatr Nephrol 7:159-162

Nesterova G, Gahl W (2008) Nephropathic cystinosis: late complications of a multisystemic disease. Pediatr Nephrol 23:863-878

Simon A, Gariepy J, Chironi G, Megnien JL, Levenson J (2002) Intima-media thickness: a new tool for diagnosis and treatment of cardiovascular risk. J Hypertens 20:159-169

Smulyan H, Asmar RG, Rudnicki A, London GM, Safar ME (2001) Comparative effects of aging in men and women on the properties of the arterial tree. J Am Coll Cardiol 37:1347-1380

Stein JH, Korcarz CE, Hurst RT et al. (2008) Use of carotid ultrasound to identify subclinical vascular disease and evaluate cardiovascular disease risk: a consensus statement from the American Society of Echocardiography Carotid Intima-Media Thickness Task Force. Endorsed by the Society for Vascular Medicine. J Am Soc Echocardiogr 21:93-111 
Town M, Jean G, Cherqui S et al. (1998) A novel gene encoding an integral membrane protein is mutated in nephropathic cystinosis. Nat Genet 18:319-324

Ueda M, O'Brien K, Rosing DR, Ling A, Kleta R, McAreavey D et al. (2006) Coronary artery and other vascular calcifications in patients with cystinosis after kidney transplantation. Clin J Am Soc Nephrol 1:555-562

Wang MC, Tsai WC, Chen JY, Huang JJ (2005) Stepwise increase in arterial stiffness corresponding with the stages of chronic kidney disease. Am J Kidney Dis 45:494-501
Wilkinson IB, MacCallum H, Flint L, Cockcroft JR, Newby DE, Webb DJ (2000) The influence of heart rate on augmentation index and central arterial pressure in humans. J Physiol 525(Pt 1):263-270

Yasmin BMJ (1999) Similarities and differences between augmentation index and pulse wave velocity in the assessment of arterial stiffness. Q J Med 92:595-600

Yilmaz MI, Qureshi AR, Carrero JJ et al. (2010) Predictors of carotid artery intima-media thickness in chronic kidney disease and kidney transplant patients without overt cardiovascular disease. Am J Nephrol 31:214-221 